\title{
Relaxation behavior, at very low frequencies, of glassy polymers containing aliphatic-aromatic side groups in their structures
}

\author{
P. Saez-Torres \\ Instituto de Ciencia y Tecnología de Polímeros (CSIC), 28006 Madrid, Spain \\ M. J. Sanchis and R. Diaz-Calleja \\ Departamento de Termodinámica, UPV, Valencia, Spain \\ J. Guzman and E. Riande ${ }^{\text {a) }}$ \\ Instituto de Ciencia y Tecnología de Polímeros (CSIC), 28006 Madrid, Spain
}

(Received 27 January 2000; accepted for publication 21 April 2000)

The curves depicting the temperature dependence of the global depolarization curves of poly(2-[naphthylacetyl)oxy]ethyl acrylate), poly(2-[naphthylacetyl)oxy]ethyl methacrylate), and the model compound of the side groups of these polymers, 2-(acetyloxy)-ethyl-(2-naphthylacetate), present ostensible glass-rubber relaxations located at $-45,34$, and $53{ }^{\circ} \mathrm{C}$, respectively. These values are very close to the respective calorimetric glass transition temperatures of these materials. As these substances exhibit a weak dielectric activity in the glassy state, it was possible to determine the components of the complex dielectric permittivity at very low frequencies from partial depolarization curves obtained on these materials in this state. Possible mechanisms responsible for the subglass relaxations are suggested from the comparison of the relaxation behavior of the model compound and the polymers. (C) 2000 American Institute of Physics. [S0021-8979(00)01515-2]

\section{INTRODUCTION}

A long-standing problem of considerable scientific and technological importance in physics of the condensed matter is the improvement of the understanding of the correlation between the chemical structure of liquids and their mechanical, dielectric, optical, etc., properties. In general, the relaxation response function of liquids and supercooled liquids to a perturbation field (mechanical, electric, etc.) describes the restructuring kinetics of the molecules of the system. ${ }^{1}$ If the relaxation response function in the time domain, $g(t)$, is normalized to unity at $t=0$, the mean-relaxation time, $\tau$, associated with the relaxation process is given by

$$
\tau=\int_{0}^{\infty} g(t) d t
$$

Although the value of $\tau$ may depend on the nature of the perturbation field, the temperature dependence of this parameter always experiences a significant increase in the vicinity of the glass-rubber transition as described by the VogelFulcher-Tammann-Hesse (VFTH) empirical equation ${ }^{2-4}$

$$
\tau=\tau_{0} \exp \left(\frac{B}{T-T_{0}}\right)
$$

where the preexponential factor $\tau_{0}$ is of the order of picoseconds. As long as the mean-relaxation time is lower than the time scale of the experiment, $\tau_{\exp }$, the liquid remains in thermodynamic equilibrium. However, as the temperature decreases, a point is reached at which $\tau>\tau_{\text {exp }}$, however large

${ }^{a}$ Electronic mail: riande@fresno.csic.es the value of $\tau_{\text {exp }}$ may be. At this temperature, known as glass transition temperature, $T_{g}$, the system falls out of equilibrium.

Upon approaching $T_{g}$, the single relaxation peak response of substances in the equilibrium liquid range and the moderately supercooled liquid regime, splits into a slow peak, called the $\alpha$ relaxation, and a fast one, named $\beta$ relaxation. The $\alpha$ relaxation displays a Kohlrausch-WilliamsWatts $(\mathrm{KWW})^{5,6}$ stretched exponential behavior given by

$$
g(t)=\exp \left[-\left(\frac{t}{\tau}\right)^{\beta}\right] ; \quad 0<\beta<1 .
$$

The $\alpha$ relaxation obeys the VFTH equation and is kinetically frozen at $T<T_{g}$ while the $\beta$ process displays an Arrhenius behavior and remains operative below $T_{g}$.

The mode coupling theory (MCT) ${ }^{7}$ has achieved considerable success in describing the $\alpha$ relaxation of the supercooled state. However, the splitting of the $\alpha \beta$ relaxation into the $\alpha$ and $\beta$ processes ${ }^{8}$ is not predicted by this theory. By postulating the existence of cooperative and noncreative regions in glass formers, a model has recently been developed ${ }^{9}$ that predicts the splitting from a high temperature single $(\alpha \beta)$ relaxation to low temperature $\alpha$ and $\beta$ processes at a certain temperature $T_{s}>T_{g}$. This model also predicts a glass transition and some anomalies in this transition which are dependent on the thermal history of the material. However, neither this model nor the MCT relate the relaxation behavior of a material to its chemical structure.

In principle, the decay function associated with the dielectric response to a perturbation field can be expressed in molecular terms by ${ }^{10}$ 


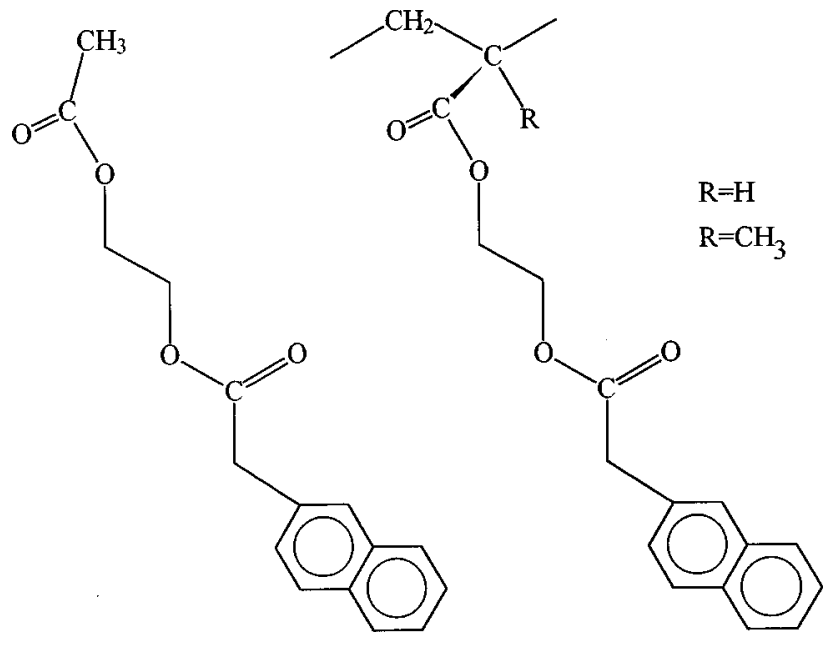

FIG. 1. Sketch of the repeating units of poly(2-[naphthylacetyl)oxy]ethyl acrylate) (PNAA) $(R=\mathrm{H})$, poly(2-[naphthylacetyl)oxy]ethyl methacrylate) (PNAMA) $\left(R=\mathrm{CH}_{3}\right)$ and the model compound 2-(acetyloxy)-ethyl-(2naphthylacetate) (ANA).

$$
g(t)=\frac{\left\langle\mu_{i}(0) \mu_{i}(t)\right\rangle+\Sigma_{i \neq j}\left\langle\mu_{i}(0) \mu_{j}(t)\right\rangle}{\left\langle\mu_{i}(0) \mu_{i}(0)\right\rangle+\Sigma_{i \neq j}\left\langle\mu_{i}(0) \mu_{j}(0)\right\rangle},
$$

where $\mu_{i}(t)$ and $\mu_{j}(t)$ are the dipole moments associated with the $i$ and $j$ relaxing dipoles, respectively. Accordingly, the determination by molecular dynamics of the trajectories of the particles within a system in the configurational space allows ${ }^{11}$ the calculation of the time-dipole correlation function $g(t)$. However, the unreasonably large computing time involved in the determination of $g(t)$ at temperatures close to the glass transition temperature restricts the use of this approach to temperatures significantly higher than $T_{g} \cdot{ }^{11} \mathrm{At}$ these temperatures, however, the $\alpha \beta$ process, and not the $\alpha$ relaxation, may be computed.

The broadness of the dielectric $\beta$ relaxation in the frequency domain suggests a variety of local environments each of them having their own barrier system. ${ }^{12}$ Different mecha- nisms associated with local molecular motions can give rise to overlapping absorptions, so that the $\beta$ relaxation appears as a single broad process. On the other hand, the lower frequency tail of the $\beta$ process may overlap with the higher frequency tail of the glass rubber relaxation thus making the resolution of this process in the supercooled liquid state complicated. This inconvenience can be circumvented by taking into account that the $\beta$ relaxation remains operative below the glass transition temperature. Therefore, the $\beta$ relaxation can be investigated in the glassy state at very low frequencies. $^{13,14}$ This approach makes the deconvolution of the $\beta$ process into its component peaks feasible, and is used in this study to investigate the dielectric activity in the glassy state of polymers containing aliphatic-aromatic flexible side groups in their structure. Subglass relaxations in polymers have traditionally been attributed to motions taking place in the side groups. In order to gain a better understanding on the mechanisms underlying the subglass relaxation, the response of poly(2-[naphthylacetyl)oxy]ethyl acrylate) (PNAA) and poly(2-[naphthylacetyl)oxy]ethyl methacrylate (PNAMA) to alternating electric fields at very low frequencies is studied using thermostimulated discharge current (TSDC) techniques. In parallel with this study, the dielectric behavior of 2-(acetyloxy)-ethyl-(2-naphthylacetate) (ANA), a model compound of the polymers side chain, is also investigated with the aim of determining the effect of the molecular connectivity on the dielectric relaxation behavior of polymers. The model compound, ANA, and the repeating units of PNAA and PNAMA are schematically represented in Fig. 1.

\section{EXPERIMENT}

2-(acetyloxy)-ethyl-(2-naphthylacetate (ANA), 2-(acryloxy)-ethyl-(2-naphthylacetate), and 2-(methacryloxy)-ethyl(2-naphthylacetate) were obtained, receptively, by adding acetyl chloride, acryloyl chloride, and methacryloyl chloride

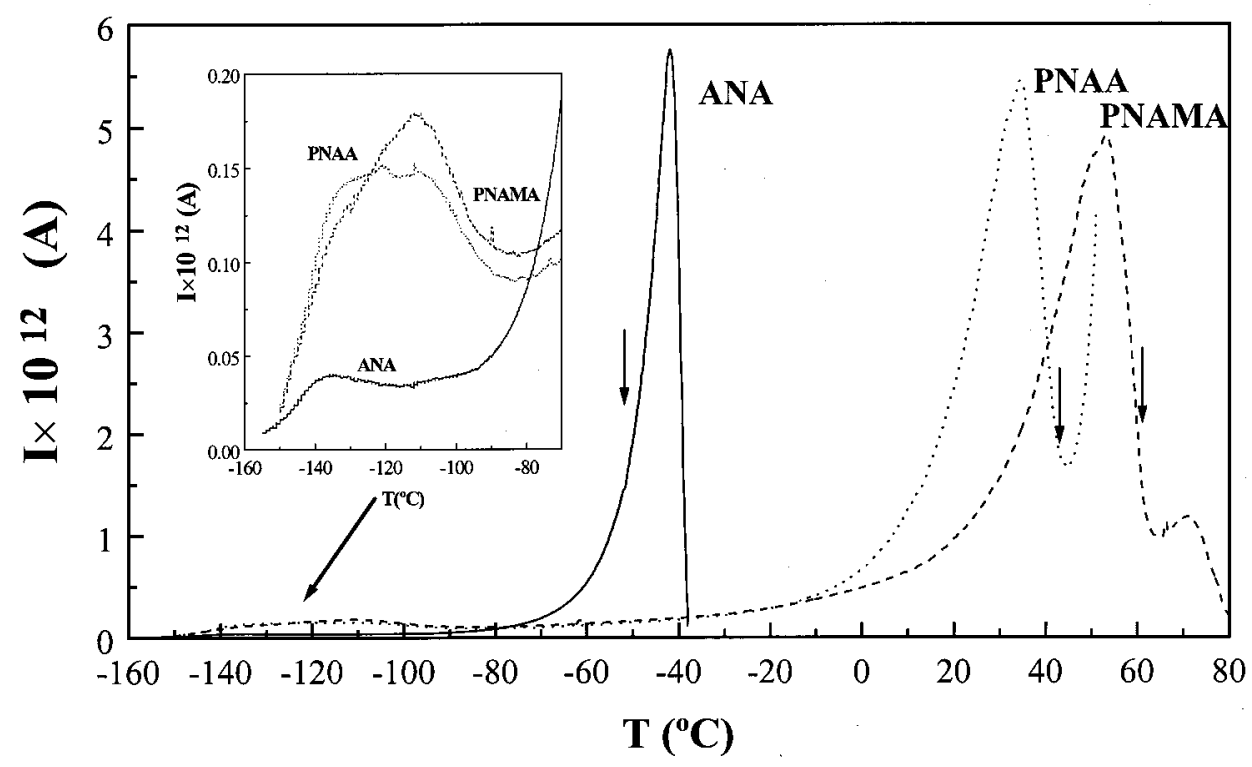

FIG. 2. Global TSDC curves for PNAA, PNAMA, and ANA. Inset: details of the curves in the glassy state. 


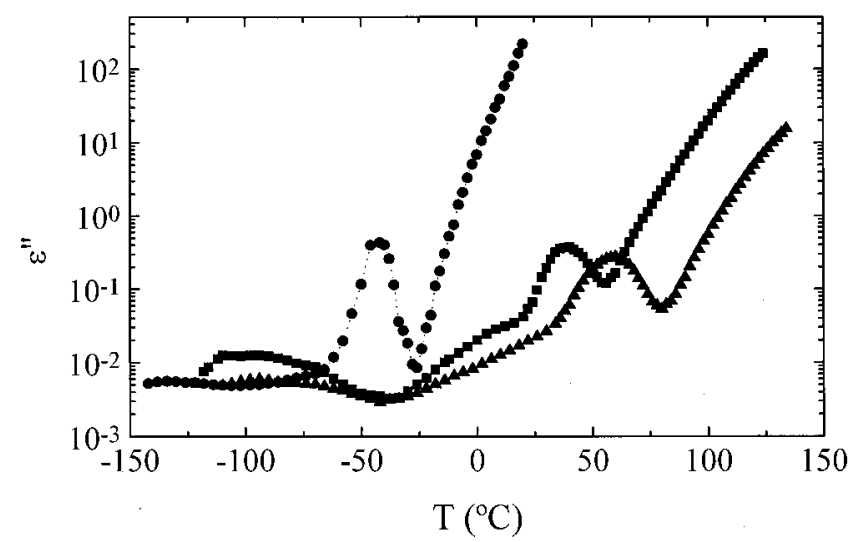

FIG. 3. Temperature dependence of the dielectric loss at $10 \mathrm{~Hz}$ for ANA $(\bullet)$, PNAA $(\diamond)$, and PNAMA $(\mathbf{\Delta})$.

to 2-hydroxyethyl-2-(2-naphthyl) acetate (HNA). Details on the synthesis of HNA and its esterification are given elsewhere. $^{15}$

Poly(2-[naphthylacetyl)oxy]ethyl acrylate) (PNAA) and poly(2-[naphthylacetyl)oxy]ethyl methacrylate) (PNAMA) were obtained in dioxane solution by the radical polymerization of 2-(acryloxy)-ethyl-(2-naphthylacetate) and 2-(methacryloxy)-ethyl-(2-naphthylacetate). The reaction was carried out at $60^{\circ} \mathrm{C}$, under vacuum, using AIBN as the initiator. The reaction was stopped after $10 \%$ of conversion was achieved. The polymers were precipitated by addition of methanol, dissolved in toluene, reprecipitated with methanol and, finally, freeze dried at room temperature in benzene solution. The glass transition temperatures of PNAA and PNAMA were measured with a Perkin Elmer DSC-7 calorimeter at a heating rate of $10{ }^{\circ} \mathrm{C} / \mathrm{min}$. The values of $T_{g}$, taken at the onset of the departure of the thermogram from the base line, were measured as $-41,34$, and $53^{\circ} \mathrm{C}$ for ANA, PNAA, and PNAMA, respectively.

Thermally stimulated discharge current curves were obtained using a TSC-RMA (Solomat) apparatus on polarized pills of $0.2 \mathrm{~mm}$ thickness. The pills, poled at $75^{\circ} \mathrm{C}$ under a potential of $500 \mathrm{~V} / \mathrm{mm}$, were quenched at $-140{ }^{\circ} \mathrm{C}$. Then the electric field was removed and the electrodes were short circuited and kept at the quenching temperature for $5 \mathrm{~min}$. The depolarization curves were obtained by heating the electrode assembly at a constant heating rate of $7^{\circ} \mathrm{C} / \mathrm{min}$. From the time derivative of the depolarization, the global discharge current curve as a function of temperature was obtained. Partial polarization discharge curves were also obtained at different temperatures in the glassy region of the materials utilized in this study, using poling windows of $10^{\circ} \mathrm{C}$.

\section{RESULTS}

The curve showing the temperature dependence of the global depolarization intensity current for the model compound and the polymers are observed in Fig. 2. The TSC spectra display an ostensible peak associated with the glassrubber transition centered at $-42,34$, and $53^{\circ} \mathrm{C}$ for ANA, PNAA, and PNAMA, respectively. The spectra exhibit a relatively weak absorption in the glassy state with the inten-

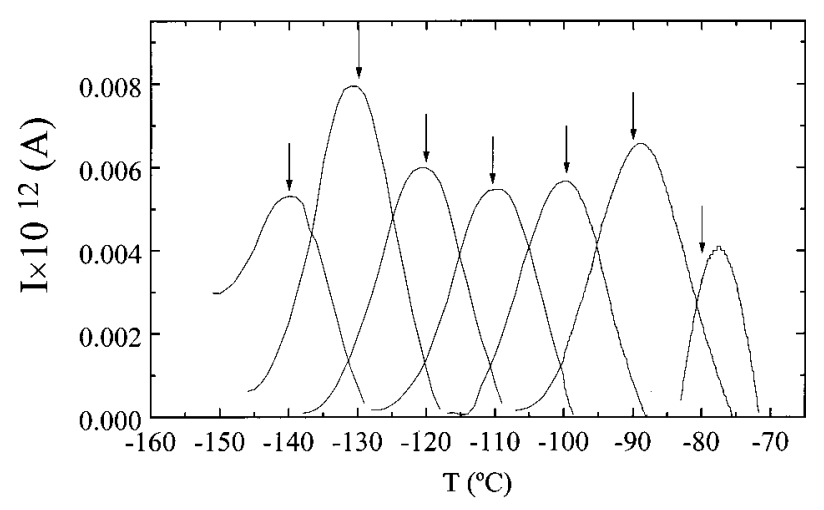

FIG. 4. Partial depolarization curves for the ANA in the glassy state. Arrows represent the polarization temperatures.

sity decreasing in the following order PNAA $>$ PNAMA $>$ ANA. The global depolarization curves do not reflect any new details in the dielectric activity of the polymers in the glassy state when compared with the ac dielectric loss curves shown in Fig. 3.

More information concerning the relaxation behavior of the polymers in the glassy state can be obtained by using partial depolarization TSC techniques. ${ }^{16}$ Partial depolarization curves obtained using the methods outlined previously are shown in Figs. 4, 5, and 6 for ANA, PNAA, and PNAMA, respectively. A close inspection of the curves corresponding to the polymers show the high dielectric activity in the region between -130 and $-90{ }^{\circ} \mathrm{C}$. On the other hand, the intensity of the elementary peaks corresponding to the model compound is significantly lower than that of the polymers.

The relaxation strength of the peaks can be obtained by taking into account that the equilibrium polarization, $P$, of a sample with cross-sectional area, $A$, under a static electric field, $E$, is given by

$$
P=\varepsilon_{0} \Delta \varepsilon E,
$$

where $\varepsilon_{0}\left(=8.854 \times 10^{-12} \mathrm{C} / \mathrm{Vm}\right)$ is the dielectric permittivity under vacuum and $\Delta \varepsilon$ is the relaxation strength at the polarization temperature of the sample. If the sample is polarized at equilibrium under an electric field, is then cooled

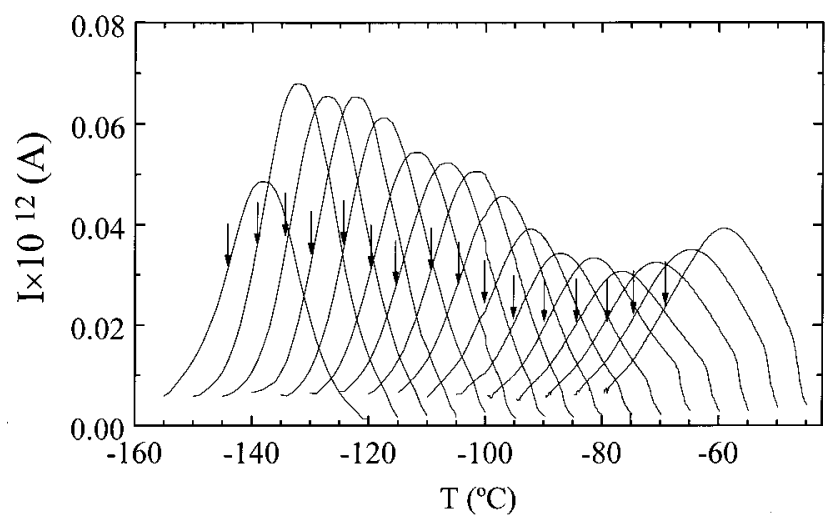

FIG. 5. Elementary depolarization curves for PNAA in the glassy state. Arrows represent the polarization temperatures. 


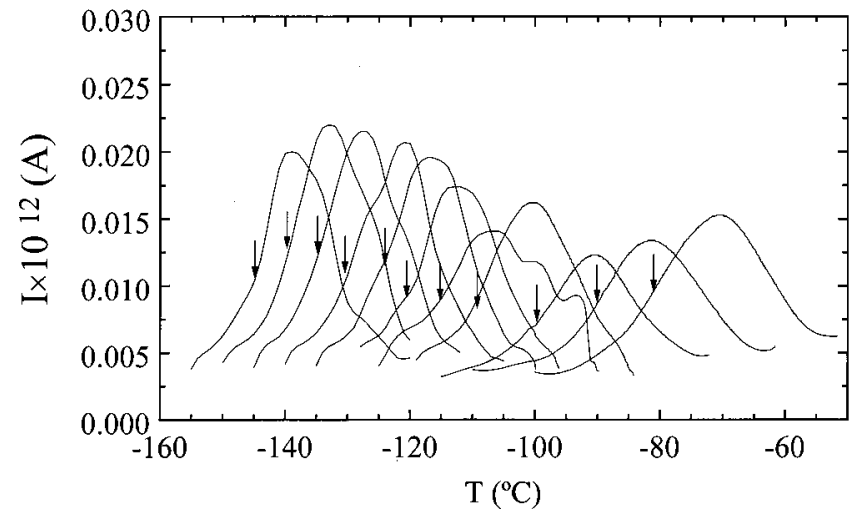

FIG. 6. Partial depolarization curves for PNAMA in the glassy state. Arrows represent the polarization temperatures.

to a temperature $T<T_{g}$, and the field is subsequently removed, the sample short-circuited and heated at a constant heating rate $h(=d T / d t)$, then the depolarization discharge current $I$ is related to the polarization by

$$
P=\frac{1}{A} \int_{0}^{\infty} I(t) d t=\frac{1}{A h} \int_{T_{0}}^{T_{f}} I(T) d t
$$

where $T_{0}$ and $T_{f}$ are, respectively, the initial and final temperatures in the interval where total depolarization takes place. Substituting this expression into Eq. (5) gives

$$
\Delta \varepsilon\left(T_{p}\right)=\frac{1}{\varepsilon_{0} E A h} \int_{T_{-0}}^{T_{f}} I(T) d T,
$$

which permits the determination of the relaxation strength associated with the poling temperatures $T_{p}$ from the integration of the corresponding partial depolarization curves. The values obtained for the model compound and the polymers are plotted as a function of the poling temperature in Fig. 7.

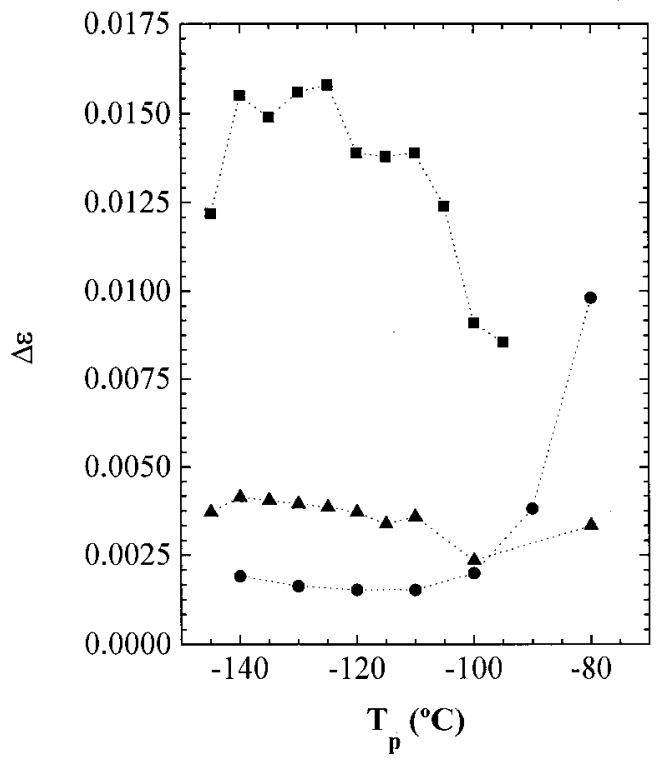

FIG. 7. Variation of the relaxation strength of the partial depolarization curves with the poling temperature for ANA $(\bullet)$, PNAA $(\diamond)$, and PNAMA $(\mathbf{\Lambda})$ in the glassy state.

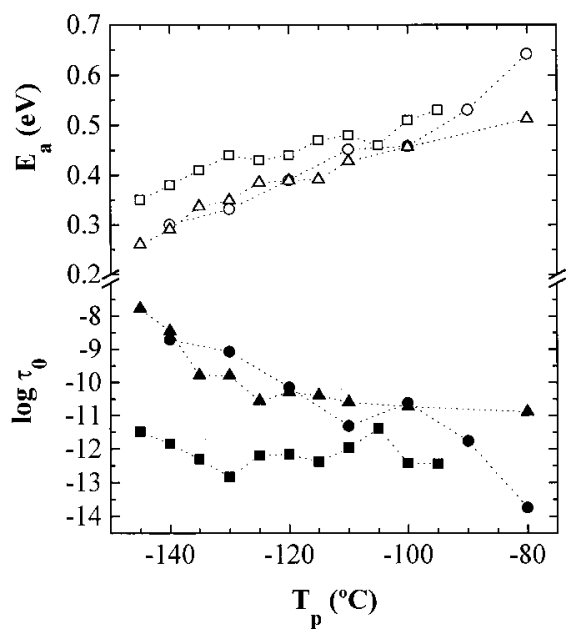

FIG. 8. Dependence of both the activation energy (open symbols) and the preexponential factor, in the Arrhenius equation, (filled symbols) on the poling temperature for the partial depolarization curves of ANA (circles), PNAA (squares), and PNAMA (triangles) in the glassy state.

The relaxation strength for PNAA shows a gradual increase with $T_{p}$, reaching a maximum at approximately $-130{ }^{\circ} \mathrm{C}$, and it then undergoes a significant decrease as the poling temperature is further increased. The relaxation strength for PNAMA is nearly independent on the poling temperature in the temperature interval $-140<T_{p}<75^{\circ} \mathrm{C}$; a similar behavior is displayed by the model compound. It is noteworthy that the relaxation strength decreases in the order PNAA $>$ PNAMA $>$ ANA.

The mean relaxation time associated with each partial depolarization curve is given by ${ }^{17}$

$$
\tau(T)=\frac{\int_{0}^{t} I\left(t^{\prime}\right) d t^{\prime}}{I(t)}=\frac{\int_{T_{i}}^{T} I\left(T^{\prime}\right) d T^{\prime}}{h I(T)},
$$

where $T_{i}$ is the initial depolarization temperature of each depolarization curve. The temperature dependence of the relaxation times calculated from Eq. (8) follows an Arrhenius behavior up to at least the half-width temperature of the peak. This allows the determination of the preexponential factors, $\tau_{0}$, and the activation energies, $E_{a}$, associated with each peak. The results for $E_{a}$ and $\tau_{0}$ are shown in Fig. 8. The values of the activation energy show a tendency to increase with increasing temperature, whereas the values of $\tau_{0}$ are nearly independent on the poling temperature for PNAA and PNAMA, and decrease with increasing values of $T_{p}$ for the model compound ANA.

A common characteristic of the relaxation behavior of the materials used in this study is the poor definition of the secondary relaxations in the ac relaxation spectra. Since the degree of overlapping of the responses associated with different mechanisms decreases with decreasing frequency, the real $\varepsilon^{\prime}$ and loss $\varepsilon^{\prime \prime}$ components of the complex dielectric permittivity $\varepsilon^{*}$ were obtained from partial TSDC curves by means of the following expression ${ }^{18,19}$ 


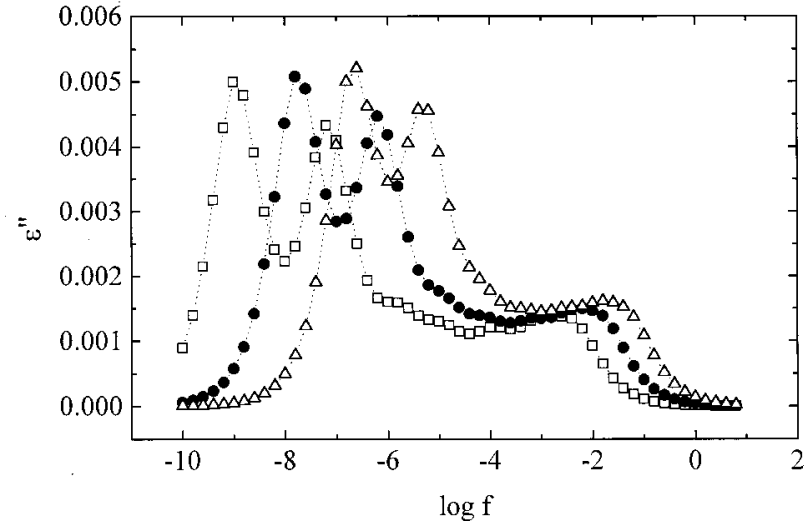

FIG. 9. The dielectric loss in the frequency domain for ANA, at different temperatures: $(\square)-100^{\circ} \mathrm{C},(\bullet)-90^{\circ} \mathrm{C}$, and $(\triangle)-80^{\circ} \mathrm{C}$.

$$
\begin{aligned}
& \varepsilon^{\prime}(\omega)=\varepsilon_{\infty}+\sum_{i=1}^{n} \frac{\left(\Delta \varepsilon_{g}\right)_{i}}{1+\omega^{2} \tau_{i}^{2}} \\
& \varepsilon^{\prime \prime}(\omega)=\sum_{i=1}^{n} \frac{\left(\Delta \varepsilon_{g}\right)_{i} \omega \tau_{i}}{1+\omega^{2} \tau_{i}^{2}} .
\end{aligned}
$$

Values of the dielectric loss in the frequency domain are represented in Figs. 9, 10, and 11 for ANA, PNAA, and PNAMA, respectively. The curves corresponding to ANA and PNAA present two well defined peaks whereas these peaks almost totally overlap in the spectrum of PNAMA. As can be seen in Fig. 12, the peaks for the model compound can be fitted to the Fuoss-Kirkwood equation ${ }^{20}$

$$
\varepsilon^{\prime \prime}(f)=\varepsilon_{\max }^{\prime \prime} \operatorname{sech}\left[m \ln \left(\frac{f\left(\varepsilon_{\max }^{\prime \prime}\right)}{f}\right)\right],
$$

where $f\left(\varepsilon_{\max }^{\prime \prime}\right)$ is the frequency at which the dielectric loss reaches its maximum value. By plotting $\cosh ^{-1}\left(\varepsilon_{\max }^{\prime \prime} / \varepsilon^{\prime \prime}\right)$ against $\ln \left[f\left(\varepsilon_{\max }^{\prime \prime}\right) / f\right]$ a straight line of a slope close to 1 was obtained for the two peaks observed in the spectra of ANA. The same analysis carried out on the relaxation spectrum of PNAA (see Fig. 13) gives a value of $m$ inferior to 1 . This would indicate that the subglass relaxation processes are

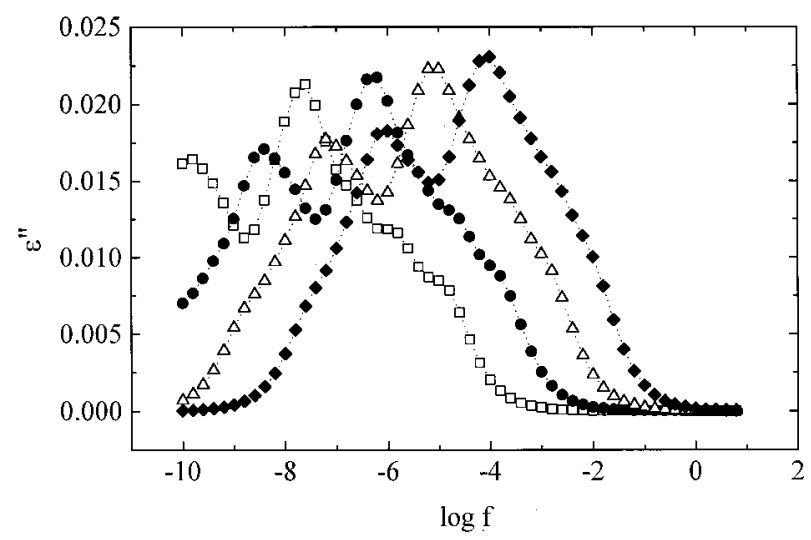

FIG. 10. Variation of the dielectric loss with frequency for PNAA, at different temperatures: $(\square)-140{ }^{\circ} \mathrm{C},(\bullet)-130{ }^{\circ} \mathrm{C},(\triangle)-120^{\circ} \mathrm{C}$, and $(\diamond)$ $-110^{\circ} \mathrm{C}$.

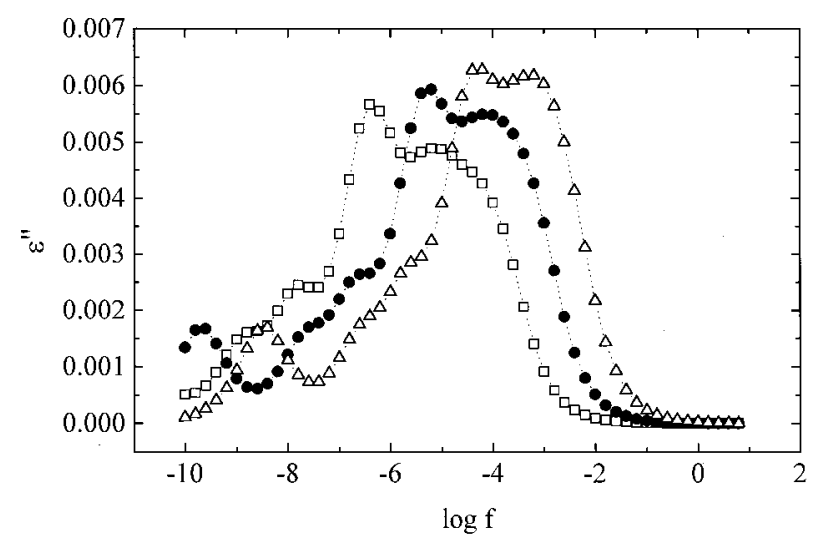

FIG. 11. The dielectric loss in the frequency domain for PNAMA, at different temperatures: $(\square)-130^{\circ} \mathrm{C},(\bullet)-120^{\circ} \mathrm{C}$, and $(\bullet)-110^{\circ} \mathrm{C}$.

more complex in this polymer than in the model compound. Furthermore, the subglass relaxation processes of PNAMA seem to be more complex than those of PNAA.

\section{DISCUSSION}

Whereas the glass-rubber relaxation arises from cooperative micro-Brownian motions of the main chain, subglass relaxations in polymers with flexible side groups seem to be related to motions taking place either in the side groups alone or combined with local motions of the main chain. ${ }^{21}$ Micro-Brownian motions involve conformational transitions that can be severely hindered by the side groups. As a result, the $\alpha$ relaxation in the frequency domain is shifted to higher temperatures as both the bulkiness and the rigidity of the side groups increase. Since the $T_{g}$ corresponds to the temperature at which the $\alpha$ relaxation becomes frozen, the substitution of hydrogen atoms in the main chain by bulky groups increases the value of $T_{g}$. For example, if the hydrogen atom of the $\alpha$ carbon of poly(methyl acrylate) is substituted by a methyl group the glass transition temperature rises from 10 to $104^{\circ} \mathrm{C}$. However, the fact that the glass transition temperature of PNAMA is only $19^{\circ} \mathrm{C}$ above that of PNAA suggests that the bulky alcohol moiety in the ester group, rather than the methyl group linked to the $\alpha$ carbon of the repeating unit, affects the micro-Brownian motion of the PNAMA chains.

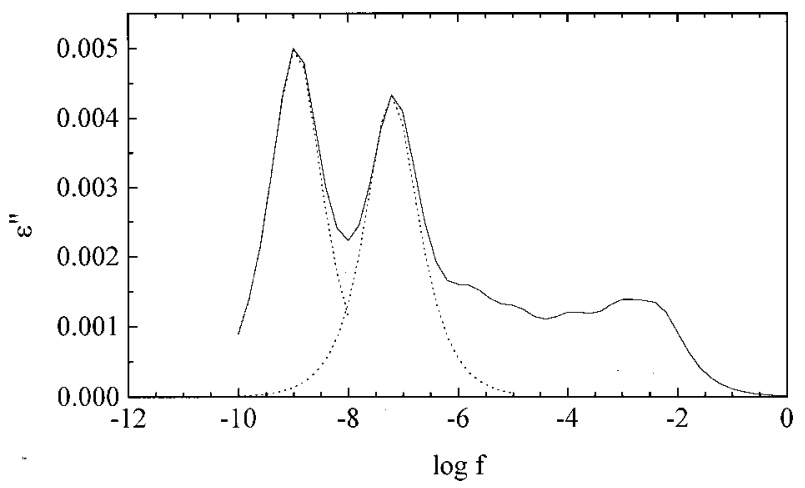

FIG. 12. Deconvolution of dielectric loss in the frequency domain, at $-100{ }^{\circ} \mathrm{C}$, for the model compound ANA. 


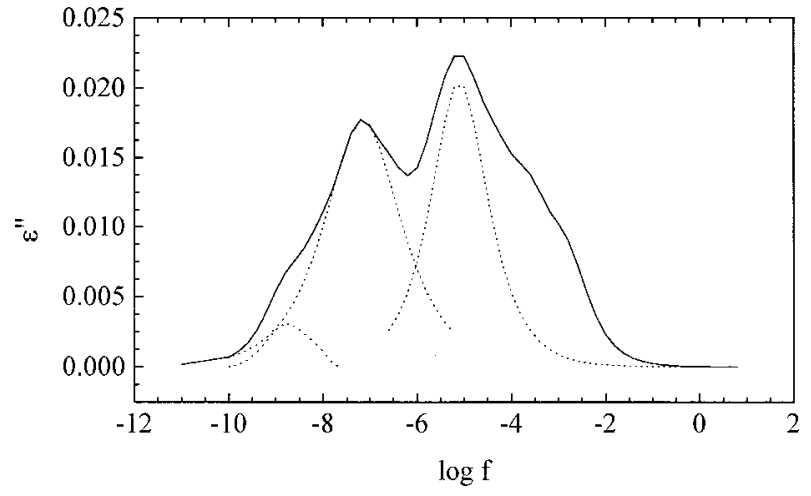

FIG. 13. Deconvolution of dielectric loss in the frequency domain for PNAA, at $-120^{\circ} \mathrm{C}$.

The polarity of the chains is customarily expressed in terms of the effective dipole moment by

$$
\mu_{\text {eff }}=\left(\frac{\left\langle\mu^{2}\right\rangle}{x}\right)^{1 / 2}
$$

where $\left\langle\mu^{2}\right\rangle$ is the mean-square dipole moment of a chain of $x$ repeating units. The experimental values of $\mu_{\text {eff }}$ are 1.91 and $2.00 \mathrm{D}$, respectively, for PNAA and PNAMA, somewhat lower than the value of $2.29 \mathrm{D}$ corresponding to the model compound. $^{22}$ The negligible subglass absorptions observed in the materials suggest that most of the dipoles relax in the glass-rubber relaxation and only a nearly negligible fraction of dipole do it in the subglass processes.

In the glassy state, the main chain of polymers is frozen so that the dipoles in the side groups relax only relative to fixed main chains and not over all configurational space as occurs in the glass-rubber relaxation. This behavior has a strong effect on the relaxation strength of the subglass processes such that its value approaches zero with decreasing temperature as the individual dipoles become confined to their lowest state energy. ${ }^{21}$ Surprisingly, in spite of the conformational wealth of the side groups in PNAA and PNAMA, the relaxation strength of the subglass processes in these polymers are rather weak, as the plots of Fig. 7 indicate. The cause of this behavior may be due to the fact that gauche states about $\mathrm{CH}_{2}-\mathrm{CH}_{2}$ bonds, which give rise to first order $\mathrm{O}-\mathrm{O}$ interactions, have an energy approximation 0.5 $\mathrm{kcal} \mathrm{mol}^{-1}$ below that of the alternative trans states. ${ }^{11,15,23} \mathrm{In}$ the same way, $\mathrm{CH}_{2}-\mathrm{O}$ bonds in the side groups prefer trans states by $\sim 1.2 \mathrm{kcal} / \mathrm{mol}^{-1}$ with respect to the alternative gauche states in which the $\mathrm{CH}_{2}-\mathrm{C}=\mathrm{O}$ bonds interact. ${ }^{11,15}$ Accordingly, most of the $\mathrm{CH}_{2}-\mathrm{CH}_{2}$ and $\mathrm{CH}_{2}-\mathrm{O}$ bonds will be located in the lowest energy states at temperatures well below $T_{g}$ and hence the contribution of motions of these bonds to the subglass relaxations will be small. These facts also would explain the low intensity subglass relaxation exhibited in the spectra of the model compound ANA.

An inspection of the dielectric loss isotherms for ANA plotted in Fig. 12 shows two well defined peaks which can be described by the Fuoss-Kirkwood equation with a value of $m$ close to 1. Each of these peaks presumably arise from conformational transitions taking place in single bonds, specifically, those of $\mathrm{CH}_{2}-\mathrm{CH}_{2}$ and $\mathrm{CH}_{2}-\mathrm{O}$ bonds. The relax- ation spectrum of PNAA in the glassy state also exhibits two peaks suggesting that motions of the $\mathrm{CH}_{2}-\mathrm{CH}_{2}$ and $\mathrm{CH}_{2}-\mathrm{O}$ bonds may also intervene in the subglass dielectric processes of this polymer. However, the deconvoluted peaks shown in Fig. 13 fit to the Fuoss-Kirkwood equation with values of $m<1$ and, as a consequence, the complexity of the subglass process in PNAA is higher than in the model compound. In this case, in addition to the motions that produce dielectric activity in ANA, conformational transitions about $\mathrm{CH}\left(\mathrm{CH}_{3}\right)-\mathrm{C}=\mathrm{O}$ bonds may be responsible for the subglass process. This hypothesis is borne out from the fact that the intensity of the subglass relaxation of PNAMA is significantly lower than that of PNAA, presumably as a consequence of the hindered motions of the $\mathrm{C}\left(\mathrm{CH}_{3}\right)-\mathrm{C}=\mathrm{O}$ bonds of the side groups by effect of the methyl groups.

\section{CONCLUSIONS}

In polyacrylates with flexible bulky side groups, the substitution of the hydrogen of the tertiary carbon in the main chain for a methyl group only increases moderately the glass transition temperature of the resulting polymer.

The dielectric relaxation spectra in the frequency domain of poly(2-[naphthylacetyl)oxy]ethyl acrylate), poly(2[naphthylacetyl)oxy] ethyl methacrylate), and the model compound of the side groups of these polymers, 2-(acetyloxy)-ethyl-(2-naphthylacetate), show a high resemblance for all samples when they are in the glassy state at very low frequencies. The fact that the two relaxations appearing in the spectra of 2-(acetyloxy)-ethyl-(2naphthylacetate) are Debye peaks suggests that they are related to conformational transitions about $\mathrm{CH}_{2}-\mathrm{CH}_{2}$ and $\mathrm{CH}_{2}-\mathrm{O}$ bonds. These mechanisms in conjunction with conformational transitions about the $\mathrm{CH}-\mathrm{CO}$ in PNAA or $\mathrm{C}\left(\mathrm{CH}_{3}\right)-\mathrm{CO}$ in PNAMA produce the subglass dielectric activity in these polymers.

Most of the bonds of the side groups are restricted in the glassy state to the conformations of lowest energy and hence the model compound and the polymers in the glassy state exhibit a low dielectric activity.

${ }^{1}$ H. Stillinger, Science 267, 1935 (1994).

${ }^{2}$ H. Vogel, Z. Phys. 22, 645 (1925).

${ }^{3}$ G. S. Fulcher, J. Am. Ceram. Soc. 8, 339 (1925).

${ }^{4}$ G. Tammann and W. Z. Hesse, Anorg. Allgem. Chem. 156, 245 (1926).

${ }^{5}$ F. Kohlrausch Pogg, Ann. Phys. (Leipzig) 91, 179 (1854).

${ }^{6}$ G. Williams and D. C. Watts, Trans. Faraday Soc. 66, 80 (1970).

${ }^{7}$ W. Götze and L. Sjögren, Rep. Prog. Phys. 55, 241 (1992).

${ }^{8}$ M. D. Ediger, C. A. Angell, and S. R. Nagel, J. Phys. Chem. 100, 13200 (1996).

${ }^{9}$ Y. N. Huang, Y. N. Wang, and E. Riande, J. Chem. Phys. 111, 8503 (1999)

${ }^{10}$ G. Williams, Dielectric Relaxation Spectroscopy of Amorphous Polymer Systems in Keynote Lectures in Polymer Science, edited by E. Riande (CSIC, Madrid, 1995)

${ }^{11}$ P. Saez-Torres, E. Saiz, R. Díaz-Calleja, J. Guzmán, and E. Riande, J. Phys. Chem. A 102, 5763 (1998).

${ }^{12}$ J. Johari, Ann. (N.Y.) Acad. Sci. 279, 117 (1976).

${ }^{13}$ R. Díaz-Calleja, M. J. Sanchis, C. Alvarez, and E. Riande, J. Appl. Phys. 81, 3685 (1997).

${ }^{14}$ R. Díaz-Calleja, M. J. Sanchis, J. Guzmán, and E. Riande, J. Appl. Phys. 84, 4436 (1998).

${ }^{15}$ P. Saez-Torres, P. Tarazona, E. Saiz, J. Guzmán, and E. Riande, J. Chem. Soc. Perkin Trans. 2, 277 (1998). 
${ }^{16}$ J. Van Turnhout, Thermally Stimulated Discharge of Polymer Electret (Elsevier, Amsterdam, 1975).

${ }^{17}$ C. Bucchi, R. Fieschi, and G. Guidi, Phys. Rev. 148, 816 (1966).

${ }^{18}$ H. Shimizu and K. Nakayama, J. Appl. Phys. 74, 1597 (1993).

${ }^{19}$ M. M. Perlman and S. Unger, J. Appl. Phys. 45, 2389 (1974).
${ }^{20}$ R. Fuoss and J. K. Kirkwood, J. Am. Chem. Soc. 63, 385 (1941).

${ }^{21}$ G. D. Smith and R. H. Boyd, Macromolecules (1990).

${ }^{22} \mathrm{P}$. Saez-Torres et al. (unpublished).

${ }^{23}$ E. Riande and E. Saiz, Dipole Moments and Birefringence of Polymers (Prentice-Hall, Englewood Cliffs, NJ, 1992). 